\title{
RNA-Seq analysis of gene expression changes triggered by Xanthomonas oryzae pv. oryzae in a susceptible rice genotype
}

\author{
Rezwan Tariq ${ }^{1 \dagger}$, Zhiyuan $\mathrm{ji}^{1,2+}$, Chunlian Wang ${ }^{1 \dagger}$, Yongchao Tang ${ }^{1}$, Lifang Zou², Hongda Sun ${ }^{1}$, \\ Gongyou Chen ${ }^{2^{*}}$ and Kaijun Zhao ${ }^{1 *}$ (D)
}

\begin{abstract}
Background: Xanthomonas oryzae pv. oryzae (Xoo) is a destructive disease in most of the rice growing regions worldwide. $X_{00}$ injects the transcriptional activator-like (TAL) effector protein into the host cell to induce the susceptibility (S) gene(s) for spreading the disease. In the current study, a susceptible rice genotype, JG30, was inoculated with wild $X_{O O}$ strain PXO99 ${ }^{\mathrm{A}}$ and its mutant $\mathrm{PH}$ without any TAL effector, to retrieve the differentially expressed genes (DEGs) having a role in susceptibility.
\end{abstract}

Results: RNA-Seq data analysis showed that 1143 genes were significantly differentially expressed ( $p$-value $\leq 0.05)$ at 12, 24, 36 and $48 \mathrm{~h}$ post inoculation (hpi). Expression patterns, evaluated by quantitative real-time PCR (qRT-PCR), of randomly selected eight genes were similar to the RNA-Seq data. KEGG pathway classified the DEGs into photosynthesis and biosynthesis of phenylpropanoid pathway. Gene ontology (GO) analysis categorized the DEGs into the biological pathway, cellular component, and molecular function. We identified 43 differentially expressed transcription factors (TFs) belonging to different families. Also, clusters of the DEGs representing kinase and peroxidase responsive genes were retrieved. MapMan pathway analysis representing the expression pattern of genes expressed highly in biotic stress and metabolic pathways after PXO99 ${ }^{\mathrm{A}}$ infection relative to $\mathrm{PH}$.

Conclusions: DEGs were identified in susceptible rice genotype inoculated with PXO99 ${ }^{\mathrm{A}}$ relative to mutant strain $\mathrm{PH}$. The identified 1143 DEGs were predicted to be included in the different biological processes, signaling mechanism and metabolic pathways. The Jasmonic acid (JA) responsive genes were identified to be

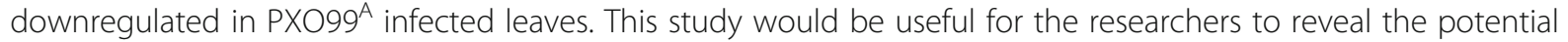
functions of genes involved in the rice susceptibility to PXO99 ${ }^{\mathrm{A}}$ infection.

Keywords: Transcriptomics, Xanthomonas oryzae, Rice, Susceptibility, Differentially expressed genes, Pathways

\section{Introduction}

Rice is a widely consumed crop, directly feeding more people than any other crop. It is the staple food in Asia, Africa and Latin America. Although rice in its various forms, has been under intense cultivation for more than 6000 years, it represents a laboratory for the investigation of bacterial diseases

\footnotetext{
*Correspondence: gyouchen@sjtu.edu.cn; zhaokaijun@caas.cn

${ }^{\dagger}$ Rezwan Tariq, Zhiyuan Ji and Chunlian Wang contributed equally to this work.

${ }^{1}$ National Key Facility for Crop Gene Resources and Genetic Improvement (NFCRI), Institute of Crop Science, Chinese Academy of Agriculture Sciences (CAAS), Beijing 100081, China

${ }^{2}$ School of Agriculture and Biology, Shanghai Jiao Tong University, 800 Dongchuan Road, Shanghai 200240, People's Republic of China
}

(Khush 1997). Currently, bacterial blight is a devastating disease in most of the rice growing regions, caused by the gram-negative bacterial pathogen, Xanthomonas oryzae pv. oryzae (Xoo) (Gnanamanickam et al. 1999). Naturally, Xoo enters inside the rice leaf through hydathodes of the leaf margin and multiplies into the intercellular spaces of epithelial tissues, then moves to the xylem vessel for systemic infection (Noda and Kaku 1999).

During infection, pathogenic Xoo injects transcriptional activator-like (TAL) effector protein via type III secretion system into host plant cell to promote the disease (Boch et al. 2009). The TALE may activate the susceptible $(S)$ gene contributing to the disease progression 
or trigger the resistance $(R)$ gene resulting to activate the host defense mechanism against pathogen. Interestingly, natural TAL effector proteins have conserved repeat region of 34 amino acids repeat, an $\mathrm{N}$ terminus region for type III secretion system, C-terminus containing acidic transcription activation domain and nuclear localization signal (Boch et al. 2009). Central conserved repeat region of TAL effectors determines the targeted genes in the host plant cell. The nucleotide specificity between TAL effector and host DNA sequence is determined by the number and order of 34 amino acids repeats (Moscou and Bogdanove 2009). Crystal structure elaborated that twelfth amino acid stabilizes the loop and thirteen amino acid residue interacts with the targeted nucleotide, projecting into the major groove (Boch and Bonas 2010; Deng et al. 2012).

Single immune receptor activates the different genes in host plant cell that need to be modulated. It was found that transcriptional reprogramming is a major feature of plant immunity and is governed by TFs and coregulatory proteins associated within discrete transcriptional complexes (Moore et al. 2011). Upon immune receptor activation, selected TFs integrate with different signaling pathways in various ways. Among all TFs, OsWRKY exhibit significant role against various diseases from seedling to adult stage of the plant through induction of the salicylic acid (SA), jasmonic acid (JA), abscisic acid and gibberellic acid pathways (Jimmy and Babu 2015). For example, OsWRKY45-2 exhibited Xoo resistance resulted in increased accumulation of JA (Tao et al. 2009); whereas, MYB TFs have promising importance in biotic resistance. Several MYB TFs have been reported with function in plant immunity, e.g., AtMYB30, AtMYB44, AtMYB108 in Arabidopsis, and HvMYB6 in barley (Ambawat et al. 2013; Chang et al. 2013). Moreover, boosting the innate immune response, plants produce different reactive oxygen species (ROS) to restrict the pathogen entry into the plant cell through the strengthening of the cell wall and cellulose deposition (Torres et al. 2006). Peroxisomes are the major sites for the accumulation of $\mathrm{H}_{2} \mathrm{O}_{2}$, as a result of the oxidative metabolism. For instance, NADPH oxidase and peroxidase class III are key proteins in ROS generation during the "oxidative burst" initiated in early defense reactions (Tripathy and Oelmüller 2012).

It is quite interesting to explore the genetic bases of the susceptibility in rice in response to the wild and mutant strains of Xoo at different time points. In the recent years, the rapid development in omics technology including transcriptomics has emerged a tremendous understanding of global gene networks to different diseases. RNA-Seq is considered unbiased technology, used to detect the DEGs with a broader dynamic range of expression level (Wang et al. 2009). The objective of the present study was to elucidate the DEGs at different time points in a susceptible rice genotype, JG30, after inoculation of the wild and mutant strains of $\mathrm{Xoo}, \mathrm{PXO}^{\mathrm{A}}$ and $\mathrm{PH}$ strains, respectively. $\mathrm{PXO}^{\mathrm{A}}{ }^{\mathrm{A}}$ was isolated in the Philippines, and it is virulent toward a large number of rice varieties representing the diverse genetic resources of resistance. Unlike PXO99 ${ }^{\mathrm{A}}, \mathrm{PH}$ is a TALE free mutant of PXO99 ${ }^{\mathrm{A}}$ (Ji et al. 2016). Comprehensive analysis of RNA-Seq data identified several DEGs; moreover, there were different clusters of the DEGs, i.e., TFs, peroxidase responsive genes etc., were retrieved, involved in different biological pathways.

\section{Results}

\section{Transcriptome profiling of JG30 genotype for DEGs}

RNA isolated from the JG30 leaves inoculated with PXO99 ${ }^{\mathrm{A}}$ and $\mathrm{PH}$ by needless syringe, were subjected to RNA-Seq (Fig. 1a); additionally, healthy leaves of JG30 plants were infected by PXO99 ${ }^{\mathrm{A}}$ and $\mathrm{PH}$ strains via scissors dipped inoculation method for confirmation of the reaction pattern; JG30 is highly susceptible to PXO99 ${ }^{\mathrm{A}}$ but not to $\mathrm{PH}$ (Fig. 1b). Summary of the RNA-Seq analysis and depicted results are given in the Additional file 1: Table S1. Raw reads were ranged from $43,590,580$ to $57,064,782$. After the low-quality reads and adapter sequences were trimmed, the clean readswere ranged from $42,414,410$ to $54,514,722$. The clean reads were mapped to the available reference genome of rice using HISAT2 (2.1.0). Approximately, 79 to $84.42 \%$ reads were successfully mapped to the rice reference genome of Nipponbare. Given the high genome coverage of the Illumina sequencing reads, we depicted that the RNA-Seq data are useful for further deep bioinformatics analysis.

To determine the expression pattern of the JG30 genotype after $\mathrm{PXO}^{\mathrm{A}}{ }^{\mathrm{A}}$ and $\mathrm{PH}$ inoculations, a pairwise comparison between $\mathrm{PXO}^{\mathrm{A}}$ vs $\mathrm{PH}$ strains was made at a specific time point. A threshold level of $p$-value was set to 0.05 and revealed 1143 significant DEGs between PXO99 ${ }^{\mathrm{A}}$ vs $\mathrm{PH}$ at different time points (Additional file 1: Table S2). An overview of the comparative analysis between PXO99 ${ }^{\mathrm{A}}$ vs $\mathrm{PH}$ revealed that there were more up-regulated genes than downregulated genes (Fig. 1c, d). Briefly, there were 263 (192 upregulated and 71 downregulated), 541 (335 upregulated and 206 downregulated), 265 (97 upregulated and 168 downregulated), and 174 (89 upregulated and 85 downregulated) DEGs were identified at 12, 24, 36 and 48 hpi in $\mathrm{PXO}^{\mathrm{A}}{ }^{\mathrm{A}}$ vs $\mathrm{PH}$.

\section{Identification of the differentially expressed TFs}

TFs are the key players, reported as differentially expressed in plants as a response to bacterial, fungal and viral infection (Amorim et al. 2017). In our experiment, 43 differentially expressed TFs, belonging to different TF families were retrieved (Fig. 2 and Additional file 1: Table S3). Among 10 differentially expressed TFs at $12 \mathrm{hpi}$, three TFs which belongs to 


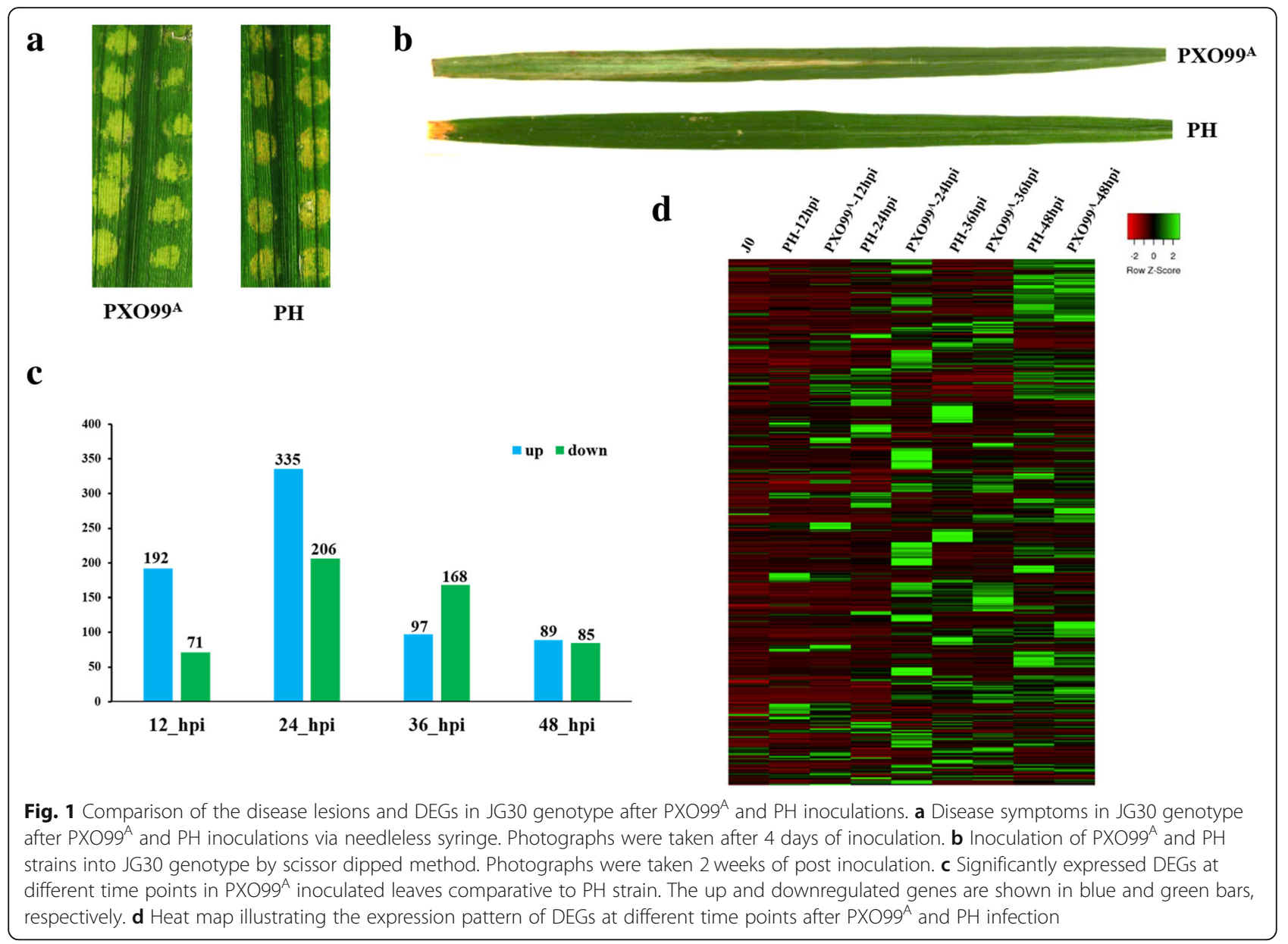

the AP2-ERF (LOC_Os03g08490.1), Dof (LOC_Os06 g17410.1) and mitochondrial-related TF (LOC_Os03g 24590.1) were found to be upregulated in PXO99 ${ }^{\mathrm{A}}$ inoculated leaves of JG30 genotype than that of PH. At $24 \mathrm{hpi}$, six AP2-ERF, two bZIP, one bHLH, two NAC, two MYB, four WRKY, and two zinc finger domain related TFs were upregulated in $\mathrm{PXO}^{\mathrm{A}}{ }^{\mathrm{A}}$ vs $\mathrm{PH}$. At $36 \mathrm{hpi}$, only two TFs belonging to the $\mathrm{C} 2 \mathrm{H} 2$ (LOC_Os07g40300.1) and heat shock (LOC_Os10g 07210.1) related TFs were upregulated; however, five TFs of different families were downregulated in PXO $99^{\mathrm{A}}$ inoculated leaves. Furthermore, four out of five differentially expressed TFs including one AP2-ERF (LOC_Os04g52090.1), two NAC (LOC_Os11g05614.1 and LOC_Os07g37920.1) and one C2H2 (LOC_Os03 g55540.1) belonged to the downregulated genes in $\mathrm{PXO}^{\mathrm{A}}$ vs PH; whereas, only one TF of AP2-ERF (L OC_Os01g04800.1) was identified to be upregulated at $48 \mathrm{hpi}$. Shortly, among $43 \mathrm{TFs}, 27$ genes were upregulated and 16 genes were downregulated in PXO99 $^{\mathrm{A}}$ inoculated leaves relative to the $\mathrm{PH}$. Hence, upregulated genes may be involved in the susceptibility after $\mathrm{PXO} 99^{\mathrm{A}}$ infection.

\section{Identification of the kinase and peroxidase responsive genes in RNA-Seq data}

Kinases form the largest gene family of the receptors in plants and have an important role in recognizing pathogen-associated molecular patterns and modulating the plant immunity in response to the invasive pathogen. We identified 28 significant differentially expressed kinase responsive genes at different time points (Fig. 3a and Additional file 1: Table S4). At 12 hpi, 10 DEGs (nine upregulated and one downregulated) were identified; among the nine upregulated DEGs, one adenylate kinase (LOC_Os11g20790.1), two MAPK (LOC_Os05g02500.1 and LOC_Os06g48590.1), one orthophosphate dikinase precursor (LOC_Os05g33570.1), two genes related to the protein kinase (LOC_Os01g48990.1 and LOC_Os0 4g52780.1), one casein kinase-like protein (LOC_Os01g5 4100.2), one CBL interacting protein kinase (LOC_Os09 g25100.1), and one wall-associated kinase (LOC_Os10g 10130.5) were retrieved to be upregulated in $\mathrm{PXO}^{\mathrm{A}}{ }^{\mathrm{A}}$ inoculated leaf samples relative to PH. In $24 \mathrm{hpi}, 10$ DEGs (nine upregulated and one downregulated) were identified. Among nine upregulated genes, including one pyrophosphate related kinase (LOC_Os01g09570.1), four 


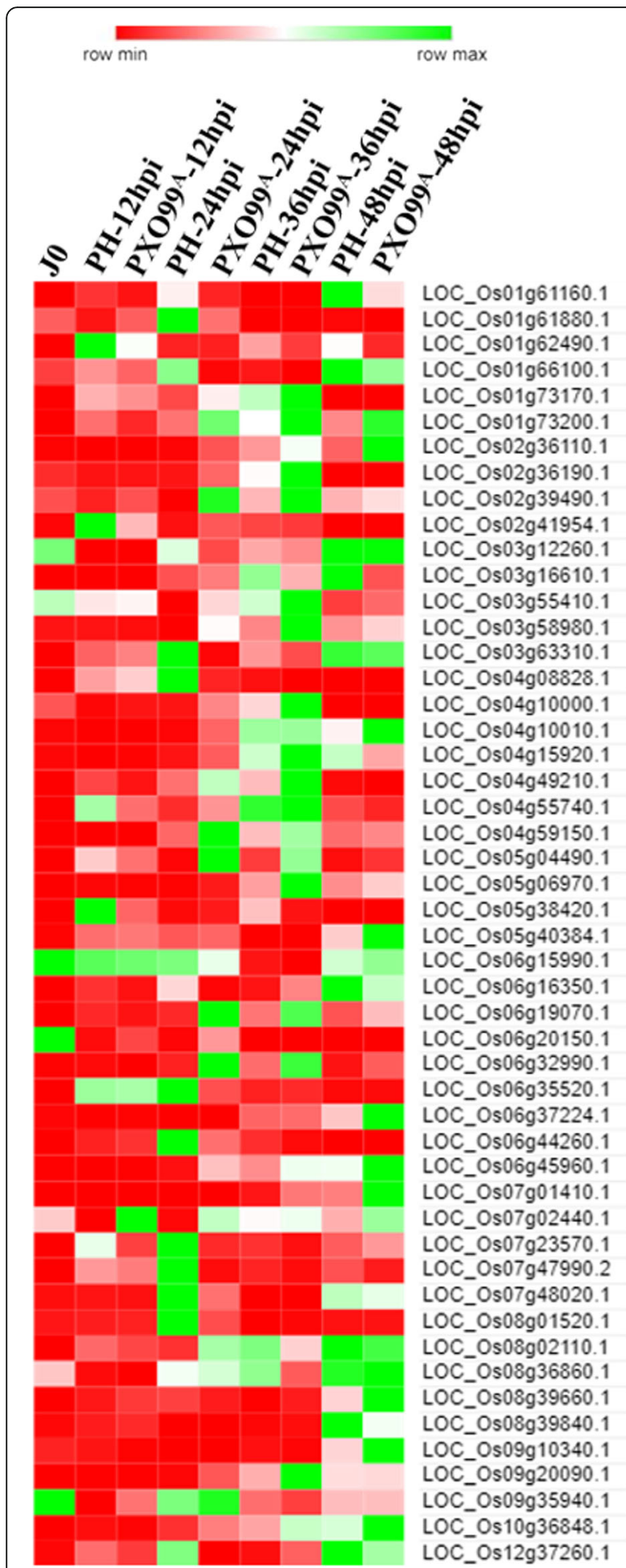

Fig. 2 Heat map exhibiting the expression patterns of differentially expressed TFs at different time intervals after $\mathrm{PXO} 99^{\mathrm{A}}$ and $\mathrm{PH}$ infection in JG30 genotype. Gene IDs and name of TFs were retrieved from RGAP and cross-checked to plant TFDB protein kinase related genes (LOC_Os02g42190.1, LOC_ Os03g50220.1, LOC_Os03g50220.1, LOC_Os09g16950.1 , and LOC_Os09g27010.1), one MAPK (LOC_Os03g1 7700.1), one KI domain interacting kinase (LOC_ Os05g41370.1), and two serine/threonine kinase related genes (LOC_Os05g46760.1 and LOC_Os02g02120.1) were upregulated in $\mathrm{PXO}^{\mathrm{A}}{ }^{\mathrm{A}}$ vs $\mathrm{PH}$. Moreover, four genes were upregulated and two genes were downregulated (LOC_Os11g12530.1 and LOC_Os11g46900.1) at 36 hpi. At 48 hpi, four genes were identified to be upregulated, including three protein kinase related genes (LOC_Os03g27990.1, LOC_Os09g18360.1, and LOC_ Os09g16950.1) and one serine/threonine kinase (LOC_ Os01g10890.1); however, one gene from phosphoenolpyruvate kinase was downregulated in $\mathrm{PXO} 99^{\mathrm{A}}$ relative to the $\mathrm{PH}$. The upregulated genes might have played role in basal defense against PXO99 ${ }^{\mathrm{A}}$, but the defense was not strong enough to cope with the pathogen attack.

The peroxidase responsive genes are important in plants controlling different processes such as development, growth, response to biotic and abiotic stress, and programmed cell death (Bailey-Serres and Mittler 2006). In our RNA-Seq experiment, 50 significant differentially expressed peroxidase responsive genes were identified at different time points (Fig. 3b and Additional file 1: Table S5). Among the 50 genes, 29 genes were upregulated and 21 genes were downregulated. At $12 \mathrm{hpi}$, two DEGs (LOC_ Os07g02440.1 and LOC_Os09g35940.1) were observed to be upregulated, and five DEGs (LOC_Os01g73200.1, LOC_ Os02g41954.1, LOC_Os04g49210.1, LOC_Os04g55740.1, and LOC_Os05g38420.1) were downregulated in PXO99 ${ }^{\mathrm{A}}$ inoculated JG30 leaves relative to PH. At $24 \mathrm{hpi}, 19$ DEGs were upregulated and 14 DEGs were downregulated in $\mathrm{PXO}^{\mathrm{A}}$ vs $\mathrm{PH}$. At $36 \mathrm{hpi}$, nine DEGs were upregulated and only two genes (LOC_Os06g15990.1 and LOC_Os08 g36860.1) were downregulated. Nevertheless, at $48 \mathrm{hpi}$, six genes (LOC_Os05g40384.1, LOC_Os06g37224.1, LOC_Os 07g01410.1, LOC_Os08g39660.1, LOC_Os09g10340.1, and LOC_Os10g36848.1) were upregulated and three genes (LOC_Os01g62490.1, LOC_Os03g16610.1, and LOC_Os08 g39840.1) were downregulated in PXO99 ${ }^{\mathrm{A}}$ inoculated leaves than that of $\mathrm{PH}$. It is depicted that downregulated peroxidase responsive genes might have role in the resistance against $\mathrm{PXO} 99^{\mathrm{A}}$ in rice.

\section{Pathway enrichment analysis}

We mapped the DEGs of JG30 genotype to the KEGG database to identify the significant pathways at different time points. The KEGG pathways were retrieved on the basis of $p$-value $\leq 0.05$ (Additional file 1: Table S6). The "biosynthesis of phenylpropanoids" and "photosynthesis" were the prominent pathways at 12,24 and $48 \mathrm{hpi}$. In photosynthesis pathway, the DEGs (LOC_Os07g37240.1, LOC_Os08g33820.1, and LOC_Os09g26810.1) were 
$\mathbf{a}$

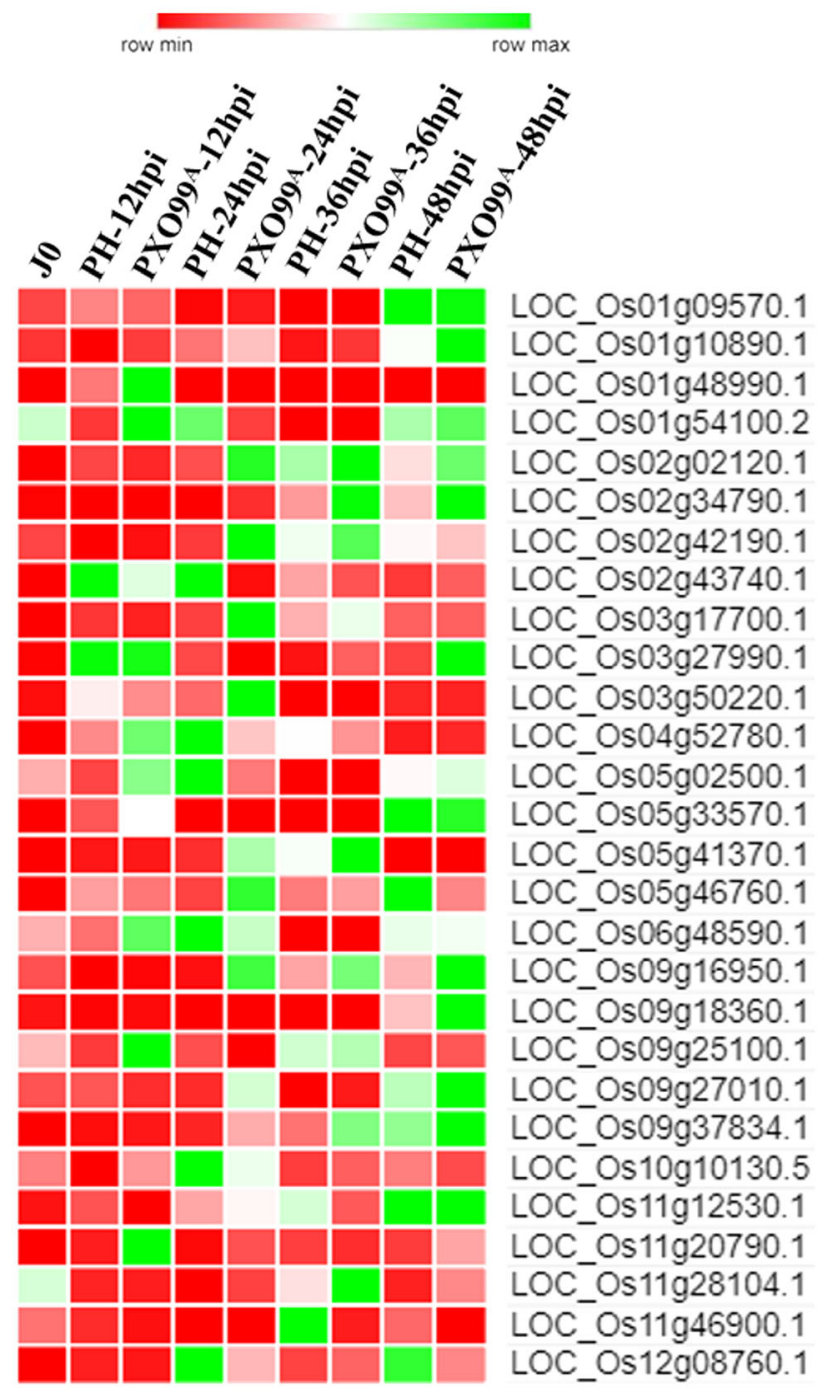

b
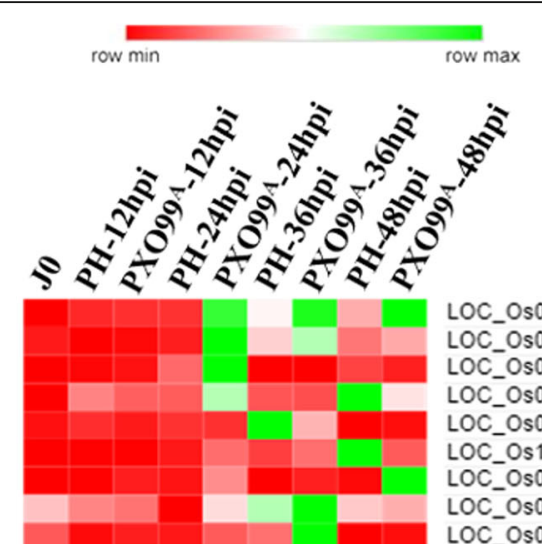

LOC_Os01g04800.1 LOC_Os05g41760.1 LOC_Os09g28440.1 LOC_Os04g52090.1 LOC_Os06g07030.1 LOC_Os11g05614.1 LOC_Os09g29820.1 LOC_Os06g41770.1 LOC_Os07g40300.1 LOC_Os02g44370.1 LOC-Os10g07210.1 LOC_Os01g11910.1 LOC_Os01g39330.1 LOC_Os09g02650.1 LOC_Os08g02070.1 LOC_Os03g24590.1 LOC_Os 12 g03040.1 LOC_Os06g51070.1 LOC_Os03g04070.1 LOC Os05g49700.1 LOC_Os02g41510.1 LOC_Os08g36920.1 LOC_Os03g08490 LOC_Os02g43820.1 LOC_Os02g52670.1 LOC_Os06g35960.1 LOC_Os01g47580.1 LOC_Os08g33150.1 LOC_Os07g37210.1 LOC_Os07g37920.1 LOC_Os11g08210.1 LOC_Os07g40130.1 LOC_Os03g08320.1 LOC_Os03g21710.1 LOC_Os01g53040.1 LOC_Os01g60640.1 LOC_Os07g48260. 1 LOC_Os03g55540.1 LOC_Os02g14990.1 LOC_Os06g17410.1 LOC_Os04g58920.1 LOC_Os06g50840.1 LOC_Os12g24490.1

Fig. 3 Heat maps demonstrating the expression patterns of the peroxidase and kinase responsive genes at different time points in JG30

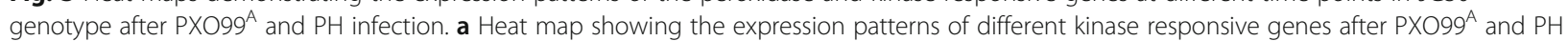
inoculations in JG30 genotype. The gene IDs were retrieved from the RGAP database. $\mathbf{b}$ Heat map of significantly expressed peroxidase responsive genes at different time points in JG30 genotype

involved in the light harvesting chlorophyll (LHC) protein complex; Lhca4 and Lhcb4 were seemed to be downregulated in PXO99 ${ }^{\mathrm{A}}$ infected leaf samples of JG30 than that of PH (Fig. 4).

To elucidate the role of identified DEGs in biotic stress response, the MapMan package was employed to investigate the genes involved in plant-pathogen interactions. The input command of the DEGs was given in MapMan package to design a particular biological process using the rice annotation project database (RAP-DB). The DEGs with known functions, e.g., TFs, secondary metabolites, ethylene, proteolysis, and signaling are shown in Fig. 5. The detailed data are given in Additional file 1: Table S7. Briefly, most of the DEGs related to the peroxidase, redox state, signaling, and MAPK responsive genes were upregulated after PXO99 ${ }^{\mathrm{A}}$ infection relative to the $\mathrm{PH}$ strain. Eight out of ten ethylene responsive genes were identified as upregulated; moreover, all the five WRKY and one Dof responsive genes were upregulated in $\mathrm{PXO} 99^{\mathrm{A}}$ infected leaf samples, which indicates that these upregulated WRKY genes might have a key role in $\mathrm{PXO}^{\mathrm{A}}{ }^{\mathrm{A}}$ infection. The expression levels of 13 DEGs representing the secondary metabolites, three pathogenesis-related (PR) genes were influenced by PXO999 


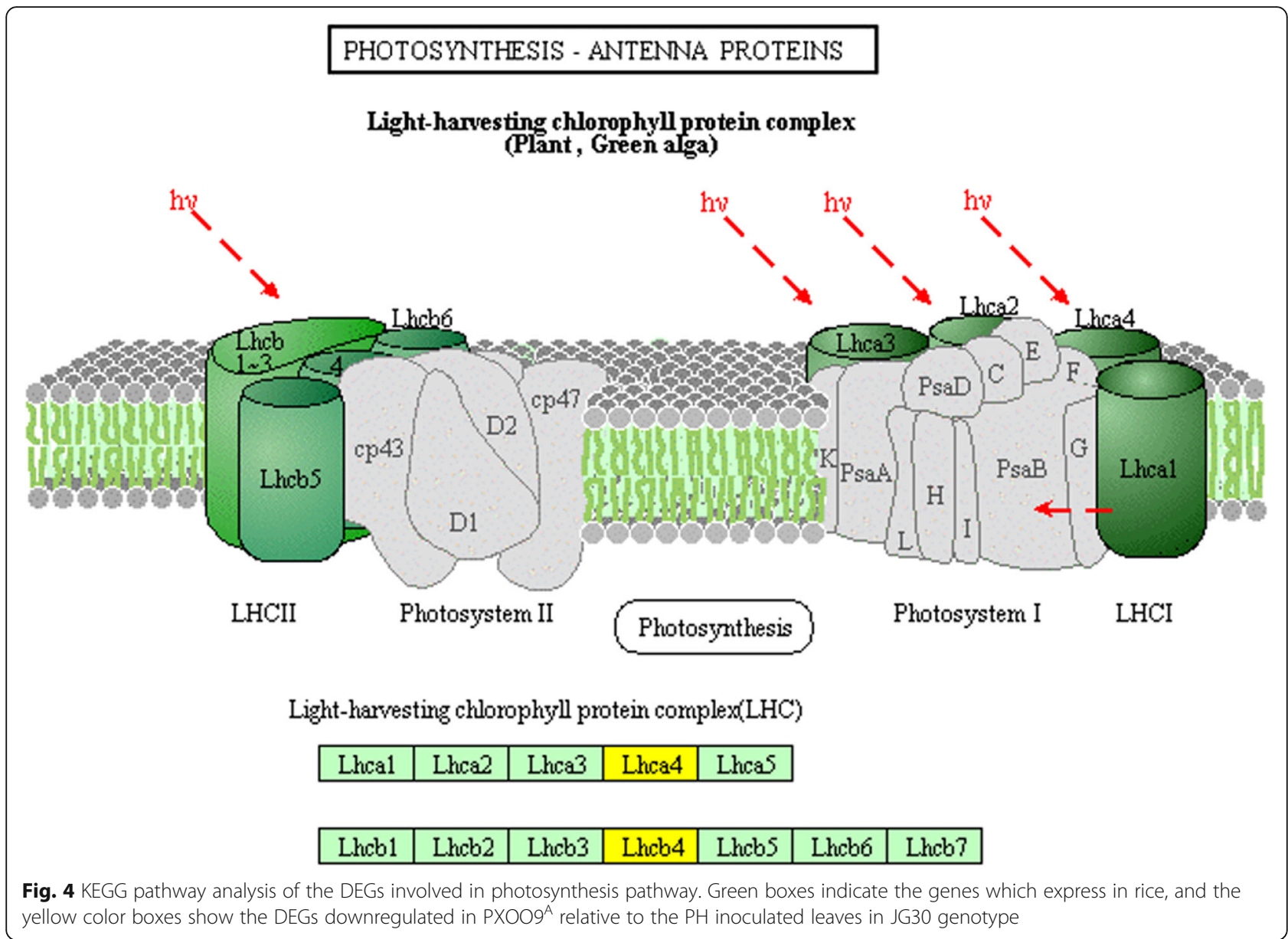

genes might have a role in the the susceptibility after PXO99 ${ }^{\mathrm{A}}$ infection in JG30 plants.

The two DEGs encoding auxins and nine ethylenerelated genes were upregulated; on the contrary, five DEGs of ethylene, three JA and one brassinosteroids responsive genes were downregulated after PXO99 $^{\mathrm{A}}$ infection. Afterward, 18 DEGs related to the cell wall were identified; among these 18 DEGs, 11 genes were upregulated and seven were downregulated in $\mathrm{PXO}^{\mathrm{A}}{ }^{\mathrm{A}}$ infected leaves as compared to the PH. Likewise, among the 31 proteolysis DEGs, 22 were upregulated and 9 nine genes were downregulated.

As an additional analysis to get a clear understanding of the participation of metabolic pathway in $\mathrm{PXO}^{\mathrm{A}}{ }^{\mathrm{A}}$ infection relative to the $\mathrm{PH}$, MapMan package was used to classify the DEGs into metabolic pathways and processes (Fig. 6 and Additional file 1: Table S8). The genes having higher expression level are involved in the "light reaction" and "photorespiration" bins representing the photosynthesis category. Additionally, some genes encoding the "lipids", "sucrose" and "starch" were upregulated in metabolic pathway. In secondary metabolism, most of the DEGs representing the "terpenes", "phenylpropanoids and phenolics", and "nucleotide metabolism" (ribonucleosidediphosphate reductase) were upregulated in susceptibility conditions after PXO99 ${ }^{\mathrm{A}}$ infection. The visual annotations of the DEGs provided a valuable resource for the exploration of the pathways involved in susceptibility after $\mathrm{PXO}^{\mathrm{A}}{ }^{\mathrm{A}}$ infection.

\section{Gene ontology enrichment analysis}

The GO analysis functionally characterizes the DEGs into three different categories, i.e., biological process, cellular component, and molecular function. The GO analysis was done using the AgriGO online tool. The GO analysis of all the DEGs is shown in the Additional file 1: Table S9. The significant GO terms after infection of $\mathrm{PXO} 99^{\mathrm{A}}$ and $\mathrm{PH}$ at each time point were retrieved using the false discovery rate (FDR) $\leq 0.05$ (Additional file 1: Table S10). In PXO99 $^{\mathrm{A}}$ vs $\mathrm{PH}$ at $12 \mathrm{hpi}$, the GO terms were significantly enriched in biological process (10), cellular component (6) and molecular function (7). At $24 \mathrm{hpi}, 56$ significant enriched GO were identified, including 28 biological processes, five cellular function, and 23 molecular function related terms. In $\mathrm{PXO}^{\mathrm{A}}{ }^{\mathrm{A}}$ vs $\mathrm{PH}$ 


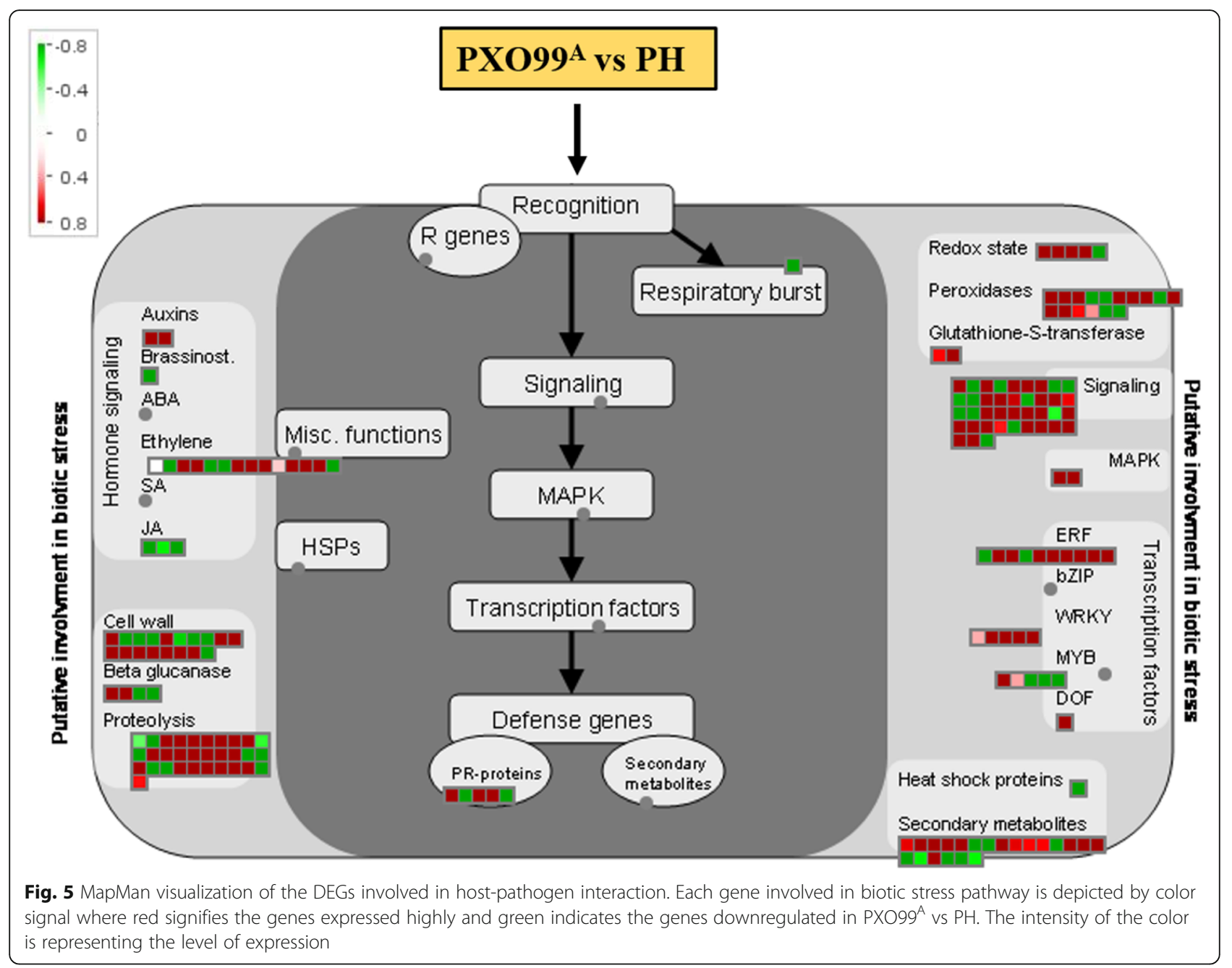

at $36 \mathrm{hpi}$, the significant GO terms were classified into the biological process (5), cellular component (1) and molecular function (13). Unlike $36 \mathrm{hpi}$, only five enriched GO terms were identified, including biological process (4) and molecular function (1).

The significant biological process related GO term in all time points $(12,24,36,48 \mathrm{hpi})$ are mentioned as follows: "biological regulation (GO: 0065007)", "response to chemical stimulus (GO: 0042221)", "response to biotic stimulus (GO: 0009607)", "lipid localization (GO:0010876)", "generation of precursor metabolites and energy (GO: 0006091)", "photosynthesis (GO:0015979)", "carbohydrate metabolic process (GO:0005975)", "response to oxidative stress (GO:0006979)", "lipid transport (GO:0006869)", "photosynthesis, light reaction (GO:0019684)", "polysaccharide metabolic process (GO:0005976)", "photosynthesis, light harvesting (GO:0009765)", and "Polysaccharide catabolic process (GO:0000272)” (Fig. 7).

\section{RNA-Seq data validation}

Eight randomly selected DEGs were evaluated for their expression patterns at different time points to validate the RNA-Seq data (Fig. 8). The gene sequences were retrieved from phytozome v12.1. LOC_Os01g03730.1, encoding nuclease I gene was highly expressed at $12 \mathrm{hpi}$ in $\mathrm{PH}$ inoculated leaves of JG30 than that of PXO99 ${ }^{\mathrm{A}}$. LOC_Os09g26810.1, representing the type II chlorophyll binding protein was upregulated at $12 \mathrm{hpi}$ in $\mathrm{PXO} 99^{\mathrm{A}}$ vs PH. Moreover, R2R3-MYB (LOC_Os02g41510.1) TF was exhibited to be overexpressed at 24 and $36 \mathrm{hpi}$ in PXO999 $^{\mathrm{A}}$ vs PH. LOC_Os04g58920.1 encoding the zinc finger domain-containing protein was induced at 24,36 and $48 \mathrm{hpi}$ in $\mathrm{PXO}^{\mathrm{A}}{ }^{\mathrm{A}}$ inoculated leaves relative to the PH. 2OG-Fe (II) oxygenase responsive gene (LOC_ Os04g49210.1) was highly expressed at 24 and $36 \mathrm{hpi}$ in $\mathrm{PXO}^{\mathrm{A}}$ vs PH, respectively. LOC_Os02g02120.1 and LOC_Os05g04490.1 representing the Serine/threonine kinase and peroxidase responsive genes, respectively, were upregulated at 24 and 36 hpi in $\mathrm{PXO}^{\mathrm{A}}$ vs $\mathrm{PH}$. 


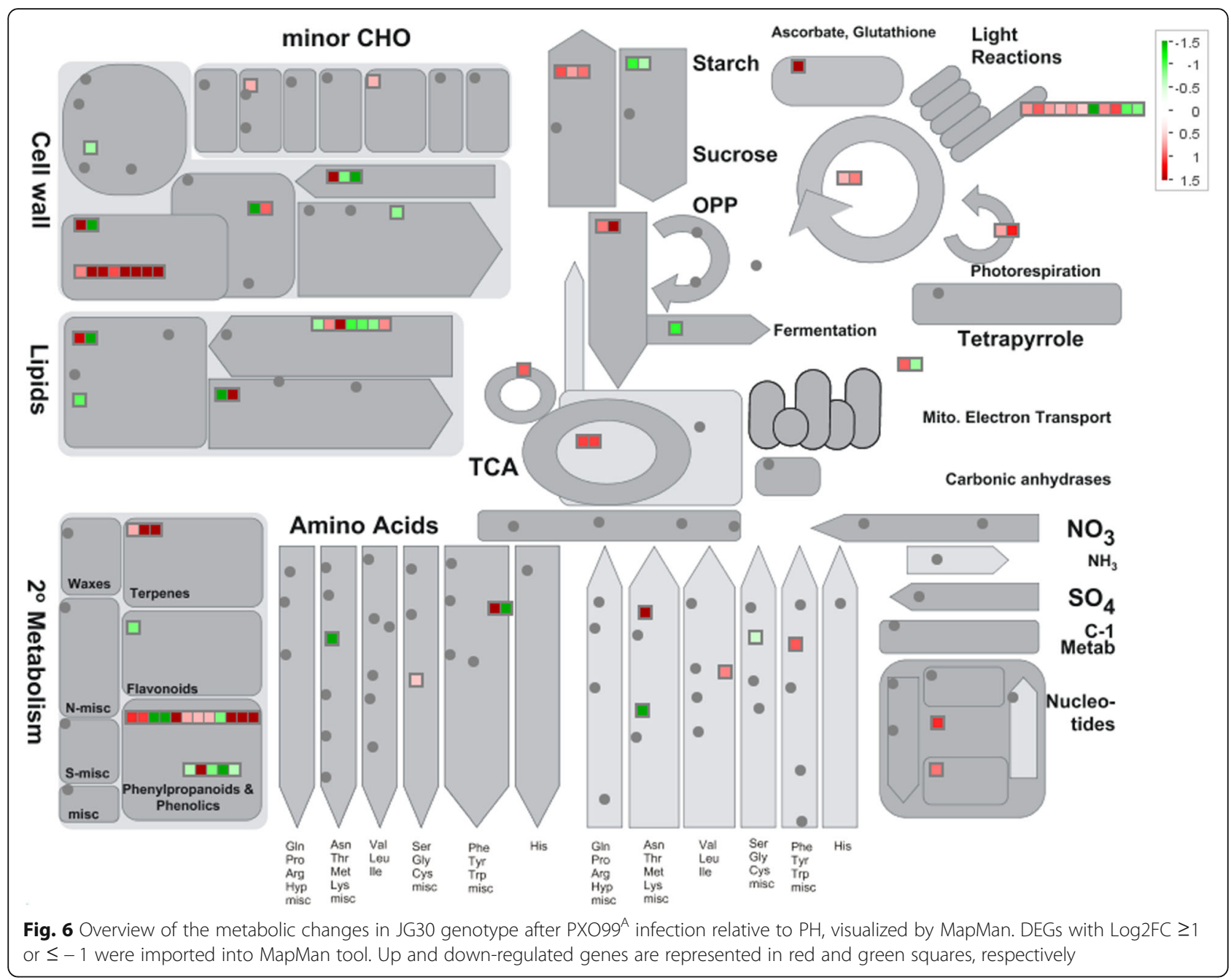

Pyruvate/Phosphoenolpyruvate kinase gene (LOC_ Os12g08760.1) was overexpressed in PH infected samples as compared to the PXO99 ${ }^{\mathrm{A}}$. In short, the qRT-PCR results validated the expression pattern of selected DEGs mentioned in RNA-Seq data.

\section{Discussion}

Plants are attacked by different pathogens that seriously damage their productivity. Plants response to these stresses by adopting different physiological, cellular and molecular mechanisms. A crucial step in plant defense against a pathogon is to timely counter the stress in a rapid and an efficient manner. In our RNA-Seq experiment, the JG30 leaves exhibited two distinct morphological symptoms after $\mathrm{PXO}^{\mathrm{A}}{ }^{\mathrm{A}}$ and $\mathrm{PH}$ infection. PXO99 ${ }^{\mathrm{A}}$ infection overcomes the PAMP-triggered immunity (PTI) owing to the fact that the secreted TAL effectors successfully interfere with or inhibit the defense response. However, the PH strain, without the TAL effector proteins, was countered by plant PTI and halted the infection. In detail, protein kinases are involved in mediating the different signaling process in the plant-pathogen interaction. The serine/threonine responsive genes (LOC_Os05g46760.1 and LOC_Os02g02120.1) were observed to be upregulated at $24 \mathrm{hpi}$ in $\mathrm{PXO} 99^{\mathrm{A}}$ vs $\mathrm{PH}$. The previous studies elaborated that serine/threonine kinase responsive genes are important in resistance against Pseudomonas syringae in tomato (Lin and Martin 2007). The most of the kinase responsive DEGs were upregulated in our experiment in susceptible leaves of JG30 after PXO99 $9^{\mathrm{A}}$ infection comparative to $\mathrm{PH}$ strain; on the contrary, kinase and signaling responsive genes are playing role in PTI as pattern recognition receptors (PPRs) and activate the plant defense system in a short time interval $(<10 \mathrm{~min})$ (Abramovitch et al. 2006; Pfeilmeier 2017); moreover, PRRs activation triggers the signaling responsive genes which further upregulate the expression of $>300$ plant genes (Cheng et al. 2017; Thilmony et al. 2006). According to the literature, to counter the PTI, pathogens deliver the effector protein into the plant cell to suppress the basal defense. In our study, PXO99 ${ }^{\mathrm{A}}$ injected the TAL effector proteins through type III secretion system into the plant cell to suppress the PTI and induce ETS. In the absence of ETI, JG30 leaves showed susceptibility 


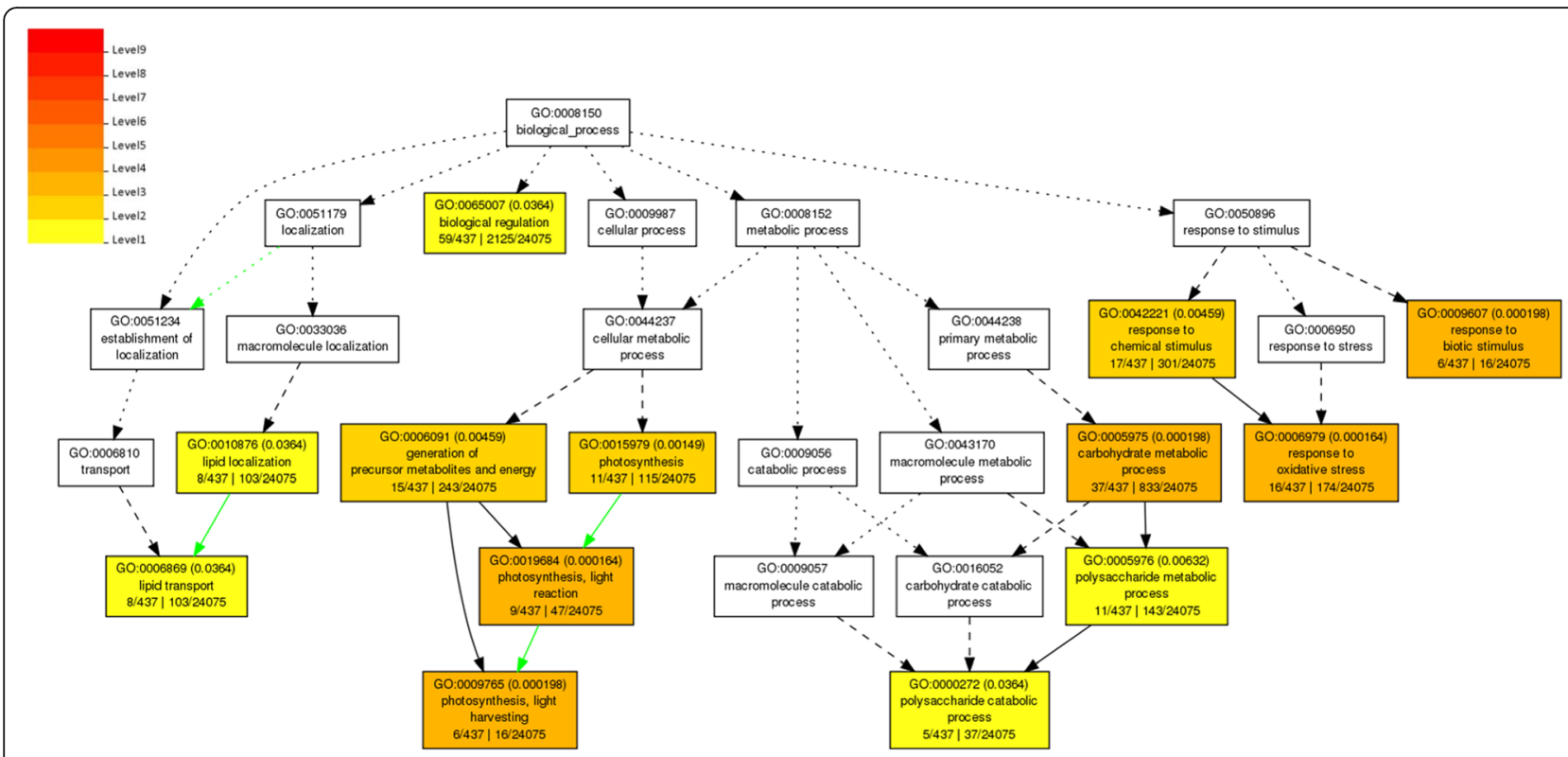

Fig. $7 \mathrm{GO}$ term of biological process exhibiting the clustering of DEGs with different coloring patterns involved in different biological processes. A key of different colors is mentioned exhibiting the significance level of the each GO term in the biological process

symptoms. Unlike PXO99 ${ }^{\mathrm{A}}$ inoculated samples, $\mathrm{PH}$ infected leaves exhibited resistance symptoms owing to the absence of effector protein in the PH strain. In a different transcriptome study, a rice genotype with Xa23 gene, exhibited the resistance against $\mathrm{PXO}^{\mathrm{A}}{ }^{\mathrm{A}}$ in form of hypersensitivity cell death reaction (Tariq et al. 2018).

Likewise, plant immune receptors activate several genes to tackle the biotic stress and coordinate their stress response with growth to maximize their fitness. TFs interact with the cis- regulatory elements in the promoter regions of stressrelated genes and up-regulate the expression of many genes to activate the biotic stress tolerance (Agarwal et al. 2010). In our experiment, OsWRKY14 (LOC_Os01g53040.1), OsWRKY17 (LOC_Os03g21710.1), and OsWRKY21 (LOC_Os01g60640.1) were upregulated in $\mathrm{PXO}^{\mathrm{A}}$ infected leaves than that of $\mathrm{PH}$. WRKY17 was exhibited to be a negative regulator of basal resistance to Pseudomonas syringae pv. tomato in Arabidopsis thaliana (Journot-Catalino et al. 2006). Whereas, overexpression of OsWRKY21 was found to be involved in the expression of calcium and calcium/calmodulin genes, important in plants as a messenger in modulating diverse physiological processes for stress adaptation (Park et al. 2005). In wheat, WRKY14 was reported to be upregulated in resistance cultivar against yellow dwarf virus infection (Wang et al. 2013).

AP2-ERF TFs are unique to plants, playing a vital role throughout the plant life cycle, e.g., leaf epidermal cell identity and response to various biotic and environmental stresses (Riechmann and Meyerowitz 1998). AP2-ERF TFs activate the defense-related genes, i.e., $P R$, osmotin, beta-1,3-glucanase and chitinase responsive genes, in Arabidopsis thaliana under different biotic stress conditions (Moffat et al. 2012; Zarei et al. 2011); similarly, ERF proteins regulate the ethylene biosynthesis pathway in Arabidopsis thaliana against Botrytis cinerea (Zhao et al. 2012). Moreover, OsERF922 was reported to negatively regulate the resistance to Magnoparthe oryzae in rice (Liu et al. 2012). AP2-ERF TFs might have regulated the $P R$ response genes in our experiment.

The different MYB responsive TFs, i.e., MYB51 (LOC_ Os08g33150.1) and R2R3-MYB (LOC_Os07g37210.1) were observed to be upregulated in the PXO99 ${ }^{\mathrm{A}}$ infected leaves of JG30. It was found that AtMYB51 is associated with wound response against insect herbivores (Johnson and Dowd 2004); The BOTRYTIS SUSCEPTIBLE1 gene encodes the MYB51, interacting with the signaling pathway in wound response, which ultimately triggers the peroxidase responsive genes (Mengiste et al. 2003). In another study, AtMYB51 was observed to regulate the indolic glucosinolate biosynthesis in leaves after pathogen attack (Gigolashvili et al. 2007). R2R3-MYB TFs control the wide variety of processes, including phenylpropanoid mechanism and secondary cell wall formation (Soler et al. 2015); in our study, R2R3-MYB TF (LOC_ Os07g37210.1) may be negatively regulating the resistance mechanism against $\mathrm{PXO} 99^{\mathrm{A}}$ infection.

The positive upregulations of the two different NAC TFs, i.e., OsNAC95 (LOC_Os06g51070.1) and OsNAC22 (LOC_Os03g04070.1) were observed in PXO99 $^{\mathrm{A}}$ infected leaves. In previous studies, OsNAC22 was observed to be overexpressed in rice infected with rice dwarf virus and rice black-streaked dwarf virus (Nuruzzaman et al. 2015). We predict 

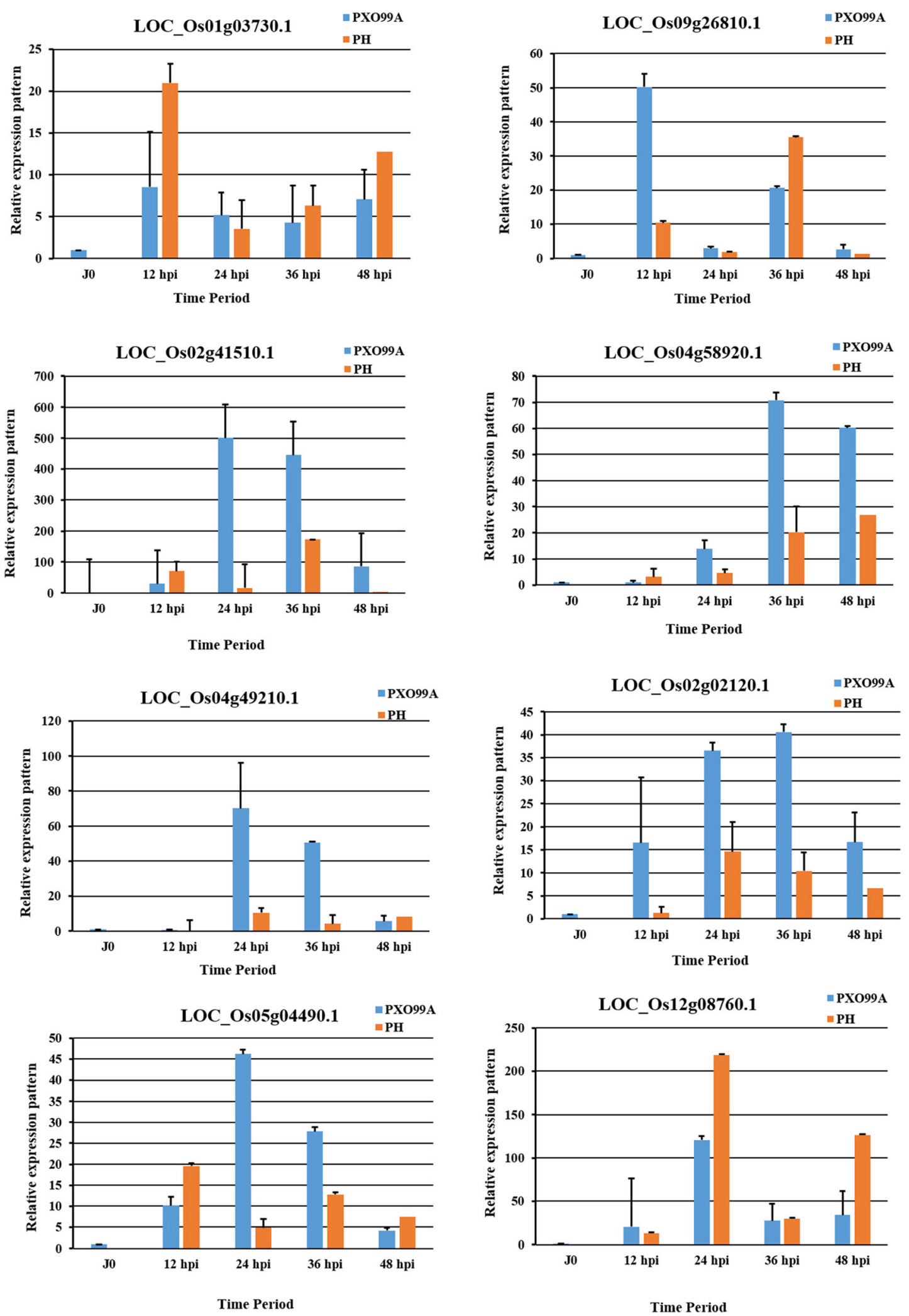

Fig. 8 qRT-PCR based expression pattern of the eight randomly selected DEGs from RNA-Seq data. Ubiquitin was used as an internal control in reaction; data are represented as mean \pm SD of three biological replicates 
that OsNAC22 might be related to the response induced by PXO99 ${ }^{\mathrm{A}}$.

Likewise, the cluster of DEGs enriched in KEGG pathway related to the photosynthesis exhibited to be downregulated after $\mathrm{PXO}^{\mathrm{A}}{ }^{\mathrm{A}}$ infection than that of $\mathrm{PH}$. Besides the KEGG pathway, the DEGs involved in the light reaction were enriched in MapMan metabolic pathway. Previous studies indicate that downregulation of the photosynthesis responsive genes reflects the usage of energy and resources to defend the invading pathogens (Jain et al. 2017; Yu et al. 2014). Pathogen infection restricts the photosynthesis activity and availability of nutrient sources for the pathogen (Berger et al. 2007); in Arabidopsis, the photosynthesis was decreased after $P$. syringae infection (Bonfig et al. 2006). Moreover, the genes involved in the photosynthesis reaction were repressed by Xoo in rice (Narsai et al. 2013). It was found in another study that Rhizoctonia solani infection leads to repressing the photosynthesis, increases the secondary metabolism, ROS accumulation and cell death in rice (Helliwell et al. 2013).

Plant hormones, JA and ET, play a diverse role in resistance against pathogens to a remarkable spectrum. In our experiment, the DEGs (LOC_Os12g37260.1, LOC_ Os08g39840.1, and LOC_Os06g11290.1) representing the JA hormones were observed to be downregulated in PXO99 ${ }^{\mathrm{A}}$ inoculated leaves. It was observed that JA regulates the expression of $P R$ genes in rice exhibiting the role in resistance to $M$. oryzae (Agrawal et al. 2000); furthermore, an increased accumulation of JA in rice was observed, causing resistance to Xoo (Tao et al. 2009). The upregulation of the ET responsive gene, OsACS2, encoding the allene oxide synthase illustrated the broad spectrum resistance to M. oryzae and $R$. solani (Helliwell et al. 2013).

The plant cell wall is the physical barrier to restrict the entry of pathogen, acting as a passive defense barrier. In our experiment, the cell wall-related genes were differentially expressed at different time points in PXO99 ${ }^{\mathrm{A}}$ vs PH. Downregulation and upregulation of the cell wall-related genes depicted to have an impact on abiotic and biotic stresses (Bacete et al. 2018). The cell wall component, lignin, was induced by different plant hormones that regulate the plant defense. In Arabidopsis, infection of $P$. syringae and X.compestris resulted in overexpression of lignin biosynthesis genes (Mohr and Cahill 2007). Similarly, hemicellulose is cell wall polysaccharides, such as xylans and xyloglucans that exhibited the resistance to Plectosphaerella cucumerina in Arabidopsis (Delgado-Cerezo et al. 2012).

Overall, the RNA-Seq data of different time points identified the DEGs. This study highlighted the genes differentially expressed only in susceptibility condition in rice after $\mathrm{PXO} 99^{\mathrm{A}}$ infection. The expression patterns of the genes expressed in susceptibility provide new information for researchers to explore the susceptibility mechanism conferred by PXO99 ${ }^{\mathrm{A}}$.

\section{Materials and methods \\ Plant materials and growth conditions}

The seeds of a rice genotype, JG30, were surface sterilized in 70\% ethanol for 5 min and washed with sterilized water. Afterward, water soaked the sterilized rice seeds for overnight. After pre-germination, rice seeds were sown in pots and kept them in the green house of Chinese Academy of Agricultural Sciences, Beijing, and P.R. China. The condition of the green house in which rice seeds were grown was: $25 / 30^{\circ} \mathrm{C}$ under a $14 \mathrm{~h}$ light $/ 10 \mathrm{~h}$ dark cycle with $80 \% \mathrm{RH}$.

\section{Inoculation of different Xoo strains}

Two different Xoo strains, PXO99 ${ }^{\mathrm{A}}$ and mutant of PXO99 $^{\mathrm{A}}(\mathrm{PH})$, were used for inoculation. Particularly, $\mathrm{PH}$ strain is without the TAL effector gene, responsible for pathogenicity symptoms in the host plant. Initially, $\mathrm{PXO}^{\mathrm{A}}$ and PH were subcultured on TSA plate (tryptophan, $10 \mathrm{~g} / \mathrm{L}$; sucrose, $10 \mathrm{~g} / \mathrm{L}$; glutamic acid, $1 \mathrm{~g} / \mathrm{L}$ and agar, $5 \mathrm{~g} / 200 \mathrm{ml}$ ) for $48 \mathrm{~h}$. The inoculum was prepared by suspending the bacterial strains in sterilized water and concentration was measured by determining the $\mathrm{OD}_{600}$ (Optical density at $600 \mathrm{~nm}$ ) between 0.9 and 1.0. The leaves of JG30 genotype were infected by using scissors dipped in bacterial suspensions to clip leaves $1-2 \mathrm{~cm}$ down from the tip of the leaf blade. After 2 weeks of post inoculation, lesions were observed from the cut surface to the distal-most position of the leaf blade exhibiting water-soaked lesions. For RNA-Seq, both strains, $\mathrm{PXO} 99^{\mathrm{A}}$ and $\mathrm{PH}$, were inoculated into 50 days old leaves of JG30 genotype by needless syringe. Inoculated leaves with three biological replicates were harvested at $12 \mathrm{hpi}$, $24 \mathrm{hpi}, 36 \mathrm{hpi}$, and $48 \mathrm{hpi}$, respectively. The harvested leaves were immediately frozen in liquid nitrogen and stored at $-80^{\circ} \mathrm{C}$ until RNA extraction.

\section{RNA extraction and Illumina sequencing}

Total RNA of inoculated (12 hpi, $24 \mathrm{hpi}, 36 \mathrm{hpi}$, and $48 \mathrm{hpi})$ and mock (J0) leaf samples were extracted through TRIZOL ${ }^{\circ}$ reagent (TIANGEN, Beijing, China) according to the manufacturer's protocol. Extracted RNA from different samples was purified by using RNase-free DNase I (TaKaRa, Kyoto, Japan) to remove the genomic DNA contamination. Total RNA concentration in different samples was calculated using NanoDrop microvolume spectrophotometer (Thermo Scientific NanoDrop Products, Waltham, MA, USA). Thereafter, the Illumina HiSeq2500 platform was used for RNA-Seq. cDNA library construction and sequencing were done by Novogene Bioinformatics Technology Co., Ltd., Beijing, China. 


\section{Analysis of the RNA-Seq data}

Quality reads of the raw RNA-Seq data were processed by the fastQC application v0.11.2 (Anders and Huber 2010); each paired-end data had the insert size 200-300 bp. The low quality reads and reads containing adapters were removed by the Trimmomatic (0.36.5) tool to get the clean reads data (Bolger et al. 2014). Afterward, paired-end clean reads were aligned to the available reference genome of rice (http://rice.plantbiology.msu.edu/ pub/data/Eukaryotic_Projects/o_sativa/annotation_dbs/ pseudomolecules/) using HISAT2 (2.1.0) (Kim et al. 2015). StringTie (1.3.4) was employed to count the number of reads mapped to each gene and quantification of the gene expression level in FPKM (number of fragments per kilobase of the transcript sequence per million base pairs sequenced) (Pertea et al. 2015). The differential expression analysis between $\mathrm{PXO} 99^{\mathrm{A}}$ and $\mathrm{PH}$ inoculated samples was performed using the DESeq2 $\mathrm{R}$ package (2.11.38). Genes with $\log 2$ fold change (Log2FC) $\geq 1$ (upregulated) or $\log 2 \mathrm{FC} \leq 1$ (down-regulated) were considered as DEGs in comparative analysis.

\section{Functional classification and pathway enrichment analysis}

The functional enrichment analysis including GO analysis was performed to identify which DEGs were significantly involved in each GO term. GO enrichment analysis was performed by AgriGO software (Du et al. 2010); GO term with FDR $\leq 0.05$ was considered significantly enriched by DEGs. The KEGG pathway analysis was executed to retrieve the enriched pathways with $p$ value $\leq 0.05$. Additionally, MapMan package was employed to get the graphical representation of the DEGs playing role in biotic stress response and metabolic pathways (Thimm et al. 2004).

\section{Validation of RNA-Seq data}

The expression pattern of the differentially expressed genes was done by qRT-PCR to validate the RNASeq data. The transcript sequences of the eight nominated genes were retrieved from Phytozome v12.1. The primers of the nominated genes were designed using AmplifX 1.5.4 software, and the primers used in the qRT-PCR were given in Additional file 1: Table S11. Ubiquitin was used as an internal control in qRT-PCR; the reaction was performed in a 96wells plate on an ABI prism 7500 Real-Time PCR system (Applied Biosystem, Foster City, CA, USA) using SYBR Green Master ROX (TaKaRa). The relative expression level of the selected DEGs was calculated with the $2^{-\triangle \Delta C T}$ method (Livak and Schmittgen 2001). The reaction was carried out using three biological replicates with three technical replicates.

\section{Conclusion}

In this study, two different strains of $\mathrm{Xoo}, \mathrm{PXO} 99^{\mathrm{A}}$ and $\mathrm{PH}$, were inoculated into the JG30 genotype leaves to uncover the differentially expressed genes at different time points. A total of 1143 genes were differentially expressed in JG30 genotype. GO and pathway analysis revealed that DEGs were involved in biological regulation, response to biotic stimulus, response to oxidative stress, lipid transport, photosynthesis, and light reaction. The antenna responsive genes having role in the photosynthesis were downregulated in $\mathrm{PXO}^{\mathrm{A}}{ }^{\mathrm{A}}$ infected leaf samples. In plant-pathogen interaction pathway, JA, brassinosteroids and ethylene responsive genes were downregulated in PXO99 ${ }^{\mathrm{A}}$ infected leaf samples than that of the PH. Moreover, most of the genes representing the cell wall and secondary metabolites were downregulated in susceptibility condition. We also identified the genes of different TF families, kinases and peroxidase responsive genes that were differentially expressed between $\mathrm{PXO}^{\mathrm{A}}$ and $\mathrm{PH}$ inoculated leaf samples. This study highlighted the possible candidate genes that may play role in susceptibility in rice after Xoo infection.

\section{Additional file}

Additional file 1: Table S1. Summary of RNA-Seq paired-end data produced by Illumina sequencing. Table S2. List of 1143 DEGs expressed at different time points in JG30 genotype after infection of PXO99 $9^{\mathrm{A}}$ and $\mathrm{PH}$ strains. The significant DEGs were retrieved by Log2FC $\geq 1$ (up-regulated genes) or $\leq-1$ (down-regulated genes) as a threshold level. The gene ID, FPKM based expression pattern, GO terms, and chromosomal position are mentioned in the table. Table S3. A detailed list of TFs expressed differentially in JG30 genotype at different time points after PXO99 ${ }^{\mathrm{A}}$ and $\mathrm{PH}$ inoculation. Table S4. List of significant enriched different Kinase responsive genes differentially expressed at different time points in JG30 genotype after $\mathrm{PXO99}{ }^{\mathrm{A}}$ and $\mathrm{PH}$ strains. Table S5. A detailed list of differentially expressed peroxidase responsive genes in JG30 genotype after PXO99 a and PH inoculations at different time points. Table S6. Significant KEGG pathways ( $P$-value $\leq 0.05$ ) involved in JG30 genotype after PXO99 ${ }^{\mathrm{A}}$ and PH infection. Table S7. MapMan analysis of DEGs involved in biotic stress pathway in JG30 genotype. The gene ID, bin code, gene description, and average Log2FC values are presented in the belowmentioned table. Table S8. MapMan analysis of DEGs involved in different metabolic pathways in JG30 genotype. The gene ID, bin code, gene description, and average Log2FC values are presented in the belowmentioned table. Table S9. Summary of GO terms enriched in JG30 genotype after PXO99 and PH infection. Table S10. A list of significantly enriched $G O$ terms ( $P$-value $\leq 0.05$ ) having DEGs at different time points after infection of $\mathrm{PXO} 99^{\mathrm{A}}$ and $\mathrm{PH}$ strains. Table S11. A list of eight genes primers used for RNA-Seq data validation. (XLSX $359 \mathrm{~kb}$ )

Acknowledgments

We thank Zheng Wei for technical assistance in inoculating the rice plants.

Authors' contributions

KZ and GC conceived and designed the research; RT, CW and LZ designed some of the experiments; RT, CW, ZJ, YT and HS performed experiments; KZ, RT and ZJ wrote the manuscript. All authors read and approved the final manuscript. 


\section{Funding}

This work was supported by grants from the National Priority ProgramBreeding New Rice Varieties for Southern China Area (2017YFD0100100), the Innovation Program of Chinese Academy of Agricultural Sciences to Kaijun Zhao and Chunlian Wang. Rezwan Tariq received support from the China Scholarship Council (CSC) for Scholarship grant.

\section{Availability of data and materials}

All the raw data of sequencing reads have been deposited in NCBI SRA database under the accession No. SAMN11081413.

\section{Ethics approval and consent to participate}

Not applicable.

\section{Consent for publication}

The manuscript has been approved by all authors.

\section{Competing interests}

The authors declare that they have no competing interests.

Received: 14 March 2019 Accepted: 24 May 2019

Published online: 24 June 2019

\section{References}

Abramovitch RB, Anderson JC, Martin GB (2006) Bacterial elicitation and evasion of plant innate immunity. Nat Rev Mol Cell Biol 7:601

Agarwal P, Agarwal PK, Joshi AJ, Sopory SK, Reddy MK (2010) Overexpression of PgDREB2A transcription factor enhances abiotic stress tolerance and activates downstream stress-responsive genes. Mol Biol Rep 37:1125

Agrawal GK, Jwa N-S, Rakwal R (2000) A novel rice (Oryza sativa L.) acidic PR1 gene highly responsive to cut, phytohormones, and protein phosphatase inhibitors. Biochem Biophys Res Commun 274:157-165

Ambawat S, Sharma P, Yadav NR, Yadav RC (2013) MYB transcription factor genes as regulators for plant responses: an overview. Physiol Mol Biol Plants 19:307-321

Amorim A, Lidiane L, da Fonseca dos Santos R, Pacifico Bezerra Neto J, GuidaSantos M, Crovella S, Maria Benko-Iseppon A (2017) Transcription factors involved in plant resistance to pathogens. Curr Protein Pept Sci 18:335-351

Anders S, Huber W (2010) Differential expression analysis for sequence count data. Genome Biol 11:R106

Bacete L, Mélida H, Miedes E, Molina A (2018) Plant cell wall-mediated immunity: cell wall changes trigger disease resistance responses. Plant J 93:614-636

Bailey-Serres J, Mittler R (2006) The roles of reactive oxygen species in plant cells. Plant Physiol 141(2):311

Berger S, Sinha AK, Roitsch T (2007) Plant physiology meets phytopathology: plant primary metabolism and plant-pathogen interactions. J Exp Bot 58:4019-4026

Boch J, Bonas U (2010) Xanthomonas AvrBs3 family-type III effectors: discovery and function. Annu Rev Phytopathol 48:419-436

Boch J et al (2009) Breaking the code of DNA binding specificity of TAL-type III effectors. Science 326:1509-1512

Bolger AM, Lohse M, Usadel B (2014) Trimmomatic: a flexible trimmer for Illumina sequence data. Bioinformatics 30(15):2114-2120

Bonfig KB, Schreiber U, Gabler A, Roitsch T, Berger S (2006) Infection with virulent and avirulent $P$. syringae strains differentially affects photosynthesis and sink metabolism in Arabidopsis leaves. Planta 225(1):1-12

Chang C, Yu D, Jiao J, Jing S, Schulze-Lefert P, Shen Q-H (2013) Barley MLA immune receptors directly interfere with antagonistically acting transcription factors to initiate disease resistance signaling. Plant Cell 25(3):1158-1173

Cheng X, Etalo DW, van de Mortel JE, Dekkers E, Nguyen L, Medema MH, Raaijmakers JM (2017) Genome-wide analysis of bacterial determinants of plant growth promotion and induced systemic resistance by Pseudomonas fluorescens. Environ Microbiol 19(11):4638-4656

Delgado-Cerezo M et al (2012) Arabidopsis heterotrimeric G-protein regulates cell wall defense and resistance to necrotrophic fungi. Mol Plant 5(1):98-114

Deng $D$ et al (2012) Structural basis for sequence-specific recognition of DNA by TAL effectors. Science 335:720-723

Du Z, Zhou X, Ling Y, Zhang Z, Su Z (2010) agriGO: a GO analysis toolkit for the agricultural community. Nucleic Acids Res 38:W64-W70

Gigolashvili T, Berger B, Mock HP, Müller C, Weisshaar B, Flügge UI (2007) The transcription factor HIG1/MYB51 regulates indolic glucosinolate biosynthesis in Arabidopsis thaliana. Plant J 50(5):886-901
Gnanamanickam S, Priyadarisini VB, Narayanan N, Vasudevan P, Kavitha S (1999) An overview of bacterial blight disease of rice and strategies for its management. Curr Sci:1435-1444

Helliwell EE, Wang Q, Yang Y (2013) Transgenic rice with inducible ethylene production exhibits broad-spectrum disease resistance to the fungal pathogens M agnaporthe oryzae and R hizoctonia solani. Plant Biotechnol J 11(1):33-42

Jain P et al (2017) Understanding host-pathogen interactions with expression profiling of NILs carrying rice-blast resistance Pi9 gene. Front Plant Sci 8:93

Ji Z, Ji C, Liu B, Zou L, Chen G, Yang B (2016) Interfering TAL effectors of Xanthomonas oryzae neutralize R-gene-mediated plant disease resistance. Nat Commun 7:13435

Jimmy JL, Babu S (2015) Role of OsWRKY transcription factors in rice disease resistance. Tropical Plant Pathol 40:355-361

Johnson ET, Dowd PF (2004) Differentially enhanced insect resistance, at a cost, in Arabidopsis thaliana constitutively expressing a transcription factor of defensive metabolites. J Agric Food Chem 52(16):5135-5138

Journot-Catalino N, Somssich IE, Roby D, Kroj T (2006) The transcription factors WRKY11 and WRKY17 act as negative regulators of basal resistance in Arabidopsis thaliana. Plant Cell 18(11):3289-3302

Khush GS (1997) Origin, dispersal, cultivation and variation of rice. Plant Mol Biol 35:25-34

Kim D, Langmead B, Salzberg SL (2015) HISAT: a fast spliced aligner with low memory requirements. Nat Methods 12(4):357

Lin N-C, Martin GB (2007) Pto-and Prf-mediated recognition of AvrPto and AvrPtoB restricts the ability of diverse Pseudomonas syringae pathovars to infect tomato. Mol Plant-Microbe Interact 20(7):806-815

Liu D, Chen X, Liu J, Ye J, Guo Z (2012) The rice ERF transcription factor OsERF922 negatively regulates resistance to Magnaporthe oryzae and salt tolerance. J Exp Bot 63:3899-3911

Livak KJ, Schmittgen TD (2001) Analysis of relative gene expression data using real-time quantitative $P C R$ and the $2-\Delta \Delta C T$ method. Methods 25:402-408

Mengiste T, Chen X, Salmeron J, Dietrich R (2003) The BOTRYTIS SUSCEPTIBLE1 gene encodes an R2R3MYB transcription factor protein that is required for biotic and abiotic stress responses in Arabidopsis. Plant Cell 15(11):2551-2565

Moffat CS, Ingle RA, Wathugala DL, Saunders NJ, Knight H, Knight MR (2012) ERF5 and ERF6 play redundant roles as positive regulators of JA/et-mediated defense against Botrytis cinerea in Arabidopsis. PLoS One 7(4):e35995

Mohr PG, Cahill DM (2007) Suppression by ABA of salicylic acid and lignin accumulation and the expression of multiple genes, in Arabidopsis infected with Pseudomonas syringae pv. Tomato. Funct Integr Genomics 7(3):181-191

Moore JW, Loake GJ, Spoel SH (2011) Transcription dynamics in plant immunity. Plant Cell 23:2809-2820

Moscou MJ, Bogdanove AJ (2009) A simple cipher governs DNA recognition by TAL effectors. Science. 326:1501-1501

Narsai R, Wang C, Chen J, Wu J, Shou H, Whelan J (2013) Antagonistic, overlapping and distinct responses to biotic stress in rice (Oryza sativa) and interactions with abiotic stress. BMC Genomics 14:93

Noda T, Kaku H (1999) Growth of Xanthomonas oryzae pv. Oryzae in planta and in guttation fluid of rice. Jap J Phytopathol 65:9-14

Nuruzzaman M et al (2015) NAC transcription factor family genes are differentially expressed in rice during infections with Rice dwarf virus, Rice black-streaked dwarf virus, Rice grassy stunt virus, Rice ragged stunt virus, and Rice transitory yellowing virus. Front Plant Sci 6:676

Park CY et al (2005) WRKY group Ild transcription factors interact with calmodulin. FEBS Lett 579(6):1545-1550

Pertea M, Pertea GM, Antonescu CM, Chang T-C, Mendell JT, Salzberg SL (2015) StringTie enables improved reconstruction of a transcriptome from RNA-seq reads. Nature Biotechnol 33(3):290-295

Pfeilmeier S (2017) Elicitation and evasion of plant innate immunity by beneficial and pathogenic bacteria. https:/ueaeprints.uea.ac.uk/66876/. Accessed 08 Feb 2019

Riechmann JL, Meyerowitz EM (1998) The AP2/EREBP family of plant transcription factors. Biol Chem 379(6):633-646

Soler M et al (2015) The Eucalyptus grandis R2R3-MYB transcription factor family: evidence for woody growth-related evolution and function. New Phytol 206(4):1364-1377

Tao Z, Liu H, Qiu D, Zhou Y, Li X, Xu C, Wang S (2009) A pair of allelic WRKY genes play opposite roles in rice-bacteria interactions. Plant Physiol 151:936-948

Tariq R et al (2018) Comparative transcriptome profiling of Rice near-isogenic line carrying Xa23 under infection of Xanthomonas oryzae pv. Oryzae. Int J Mol Sci 19:717

Thilmony R, Underwood W, He SY (2006) Genome-wide transcriptional analysis of the Arabidopsis thaliana interaction with the plant pathogen Pseudomonas 
syringae pv. Tomato DC3000 and the human pathogen Escherichia coli O157: H7. Plant J 46:34-53

Thimm O et al (2004) MAPMAN: a user-driven tool to display genomics data sets onto diagrams of metabolic pathways and other biological processes. Plant J 37:914-939

Torres MA, Jones JD, Dangl JL (2006) Reactive oxygen species signaling in response to pathogens. Plant Physiol 141:373-378

Tripathy BC, Oelmüller R (2012) Reactive oxygen species generation and signaling in plants. Plant Signal Behav 7(12):1621-1633

Wang X, Liu Y, Chen L, Zhao D, Wang X, Zhang Z (2013) Wheat resistome in response to barley yellow dwarf virus infection. Funct Integr Genomics 13(2):155-165

Wang Z, Gerstein M, Snyder M (2009) RNA-Seq: a revolutionary tool for transcriptomics. Nat Rev Genet 10(1):57-63

Yu C, Chen H, Tian F, Leach JE, He C (2014) Differentially-expressed genes in rice infected by Xanthomonas oryzae pv. Oryzae relative to a flagellin-deficient mutant reveal potential functions of flagellin in host-pathogen interactions. Rice 7:20

Zarei A, Körbes AP, Younessi P, Montiel G, Champion A, Memelink J (2011) Two GCC boxes and AP2/ERF-domain transcription factor ORA59 in jasmonate/ ethylene-mediated activation of the PDF1. 2 promoter in Arabidopsis. Plant Mol Biol 75:321-331

Zhao Y, Wei T, Yin KQ, Chen Z, Gu H, Qu LJ, Qin G (2012) Arabidopsis RAP2. 2 plays an important role in plant resistance to Botrytis cinerea and ethylene responses. New Phytol 195:450-460

\section{Publisher's Note}

Springer Nature remains neutral with regard to jurisdictional claims in published maps and institutional affiliations.

\section{Submit your manuscript to a SpringerOpen ${ }^{\odot}$ journal and benefit from:}

- Convenient online submission

- Rigorous peer review

- Open access: articles freely available online

High visibility within the field

- Retaining the copyright to your article

Submit your next manuscript at $\boldsymbol{\nabla}$ springeropen.com 